\title{
Correction
}

\section{Correction to: Stein and Weinstein structures on disk cotangent bundles of surfaces}

\author{
BuraK OzBagCi(iD
}

\section{Correction to: Arch. Math. \\ https://doi.org/10.1007/s00013-019-01357-y.}

After our article entitled "Stein and Weinstein structures on disk cotangent bundles of surfaces" was accepted and appeared online, it was pointed out by Sylvain Courte that our proof of the existence of the symplectomorphisms in Theorem 1.1 is flawed. Our proof of the existence of the contactomorphisms, however, holds true in both parts of Theorem 1.1.

The main result of [2] should be restated as follows:

Theorem 0.1. (a) Suppose that $\Sigma_{g}$ is a closed, connected, smooth, and orientable surface of genus $g \geq 1$. Then, the boundary of the Stein handlebody diagram depicted in $\left[2\right.$, Figure 1] is contactomorphic to $\left(\mathbb{S} T^{*} \Sigma_{g}, \xi_{\text {can }}\right)$.

(b) Suppose that $N_{k}$ is a closed, connected, smooth, and nonorientable surface of genus $k \geq 1$, i.e., $N_{k}=\#_{k} \mathbb{R P}^{2}$. Then, the boundary of the Stein handlebody diagram depicted in [2, Figure 2] is contactomorphic to $\left(\mathbb{S} T^{*} N_{k}, \xi_{\text {can }}\right)$.

Proof. The proof of (a) is given in [2, Remark 3.2] and the proof of (b) holds true as given in [2, Section 3.2].

In [2, Theorem 1.1], we also claimed that the Stein domain depicted in $\left[2\right.$, Figure 1] is symplectomorphic to $\left(\mathbb{D} T^{*} \Sigma_{g}, \omega_{\text {can }}\right)$ and the Stein domain depicted in [2, Figure 2] is symplectomorphic to $\left(\mathbb{D} T^{*} N_{k}, \omega_{\text {can }}\right)$. Our proof (in both cases) is flawed since first of all we misquoted [1, Theorem 13.8] as Theorem 3.1 in our paper. The conclusion of [1, Theorem 13.8] is the existence of an isotopy of embeddings $h_{t}: V \rightarrow V$ with $h_{0}=I d$, and satisfying certain properties but in [2, Theorem 3.1], we inadvertently quoted this part as the existence of a diffeomorphism $h: V \rightarrow V$ isotopic to the identity. Regardless of this error, we now realized that [1, Theorem 13.8] does not make

The original article can be found online at https://doi.org/10.1007/s00013-019-01357-y. 
any statement about relating a Weinstein structure to a given Stein structure, but only about realizing a Weinstein structure as a Stein structure within a given complex manifold. Therefore, although the existence of the aforementioned symplectomorphisms is plausible, it seems impossible to prove it using [1, Theorem 13.8].

Finally, we would like to emphasize that the discussion about the surgery diagrams for the canonical contact structures in Section 4 of our article [2] is unaffected by this error.

Publisher's Note Springer Nature remains neutral with regard to jurisdictional claims in published maps and institutional affiliations.

\section{References}

[1] Cieliebak, K., Eliashberg, Y.: From Stein to Weinstein and Back. Symplectic Geometry of Affine Complex Manifolds. American Mathematical Society Colloquium Publications, 59. American Mathematical Society, Providence, RI (2012)

[2] Ozbagci, B.: Stein and Weinstein structures on disk cotangent bundles of surfaces. Arch. Math. (2019). https://doi.org/10.1007/s00013-019-01357-y

\section{Burak OzBagci}

Department of Mathematics

Koç University

Istanbul

Turkey

e-mail: bozbagci@ku.edu.tr 